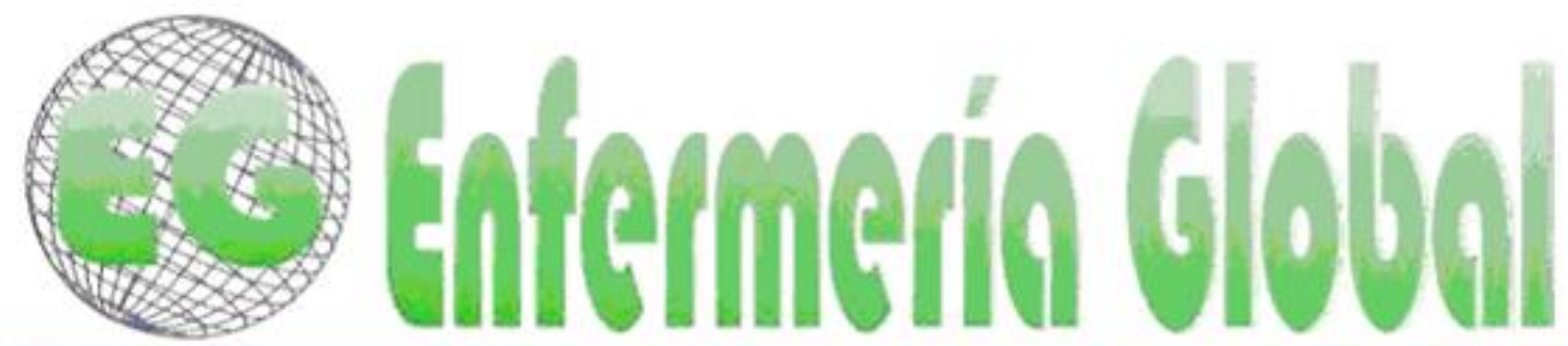

\title{
CLÍNICA
}

\section{Estudio sobre la resiliencia en ancianas del Valle de Paraíba, São Paulo, Brasil}

Estudo sobre a resiliência em idosas do Vale do Paraíba, São Paulo, Brasil

Study of resilience in the elderly Paraíba Valley, Sao Paulo, Brazil

\section{*Araújo, Claudia Lysia de Oliveira **Faro, Ana Cristina Mancussi}

\author{
*Profesor de la Facultad Integrada Teresa de Ávila - Lorena, São Paulo, Brasil. Grupo de Investigación \\ en Rehabilitación, funcionalidad y educación sanitaria. E-mail: claudialysia@usp.br **Profesor Titular \\ de Departamento de Enfermería Médico-Quirúrgica de la Universidad de São Paulo Escuela de \\ Enfermería. Advisor. Grupo de Investigación en Rehabilitación, Funcionalidad y Educación para la \\ Salud.
}

Palabras clave: envejecimiento; resiliencia; salud de la mujer; enfermería; gerontología. Palavras chave: envelhecimento; resiliência; saúde da mulher; enfermagem; gerontologia Keywords: aging; resistance; women's health; Nursing; gerontology

\section{RESUMO}

A resiliência é o termo que vem da física, muito discutido em várias áreas, diante deste referencial, o objetivo deste foi analisar a resiliência de mulheres idosas, participantes de atividades no Centro de Convivência. Estudo exploratório, descritivo, transversal, com abordagem quantitativa, caracterizou-se 117 mulheres com idade média de 70-80 anos, viúvas, católicas, aposentadas, de 1-5 anos de educação escolar, com renda familiar de 2-3 salários mínimos, praticantes de atividade física, composição familiar de 1-2 membros na família, com percepção positiva para os cuidados com a saúde física e mental, em uso de 1-2 tipos de medicamentos por dia, apresentam 1-2 comorbidades, relatam atividade sexual 1- 4 vezes por mês e com nível de resiliência elevado

\section{RESUMEN}

La resiliencia es un término que proviene de la física, muy discutido en varias áreas, antes de este referencial, el objetivo de este estudio fue analizar la resiliencia de las mujeres mayores, participantes en las actividades en el Centro de Convivencia. Estudio exploratorio, descriptivo, transversal, con abordaje cuantitativo, caracterizado por 117 mujeres con una edad media de 70 a 80 años, viudas, católicas, jubiladas, de 1-5 años de educación escolar, con ingreso familiar de 2-3 salarios mínimos, practican actividad física, composición familiar de 1-2 miembros en la familia, con percepción positiva para el cuidado de la salud física y mental, toman de 1-2 tipos de medicamentos al día, presentan 1-2 comorbilidades, relatan actividad sexual 1 - 4 veces al mes y con nivel de resiliencia elevado 


\section{ABSTRACT}

Resilience is a term coming from physics that has been discussed in many areas prior to this paper. The aim of this study is to analyse resilience of elderly women taking part in the activities of the Centre for the Elderly. It is an exploratory, descriptive, cross-functional study with a quantitative approach. It is conducted on 117 women with an average age between 70-80 years. These women have different characteristics: they are widows, Catholics, retired, have 1-5 years of school education, with a family income based on 2-3 minimum wages, they exercise, they have a family made up of 1-2 members, they have a positive perception of physical and mental health care, take 1-2 types of medicines a day, suffer from 1-2 comorbidities, they report being sexually active1-4 times a month and have a high level of resilience.

\section{INTRODUCCIÓN}

El envejecimiento de la población mundial es un fenómeno nuevo al que incluso los países desarrollados todavía están tratando de adaptarse. Lo que fue en el pasado una marca de unos pocos países se ha convertido en una experiencia de crecimiento en todo el mundo.

Siguiendo los patrones observados en otros países ${ }^{(1)}$, en Brasil, la disminución de la fecundidad y el aumento de la esperanza de vida resultaron en los últimos 30 años en aumento significativo de la población de edad avanzada. Además, la mejora relativa en el acceso de la población a los servicios de salud, campañas de vacunación nacionales, aumentando el nivel educativo de la población, las inversiones en infraestructura de saneamiento y la percepción de las personas en relación con la enfermedad forman un conjunto de factores que contribuyen al aumento de la esperanza de vida de los ancianos. Del mismo modo, la alta fecundidad observada en el siglo XX, en los años 50 y 60, y la disminución de la mortalidad que benefició a todos los grupos de población condujo al diseño de este perfil demográfico ${ }^{(2,3)}$.

Una de las características de envejecimiento de la población en Brasil es la pobreza. Las pensiones son la principal fuente de ingresos de la población de edad avanzada, pero sus valores bajos son insuficientes para la provisión de todas las necesidades de las personas mayores que residen en la comunidad, sobre todo cuando se ven afectadas por una o más enfermedades crónicas que causan deterioro de su independencia. La inversión pública en apoyo a las personas mayores y cuidadores, ya sea a través de ayudas financieras, ya sea ofreciendo servicios alternativos como hospitales de día y centros de día, o el apoyo en áreas como la alimentación, el transporte y el cuidado de la salud, entre otros, es muy bajo ${ }^{(4)}$.

Brasil actualmente tiene una población de más de 190 millones de personas y $56 \%$ de mujeres. La esperanza de vida femenina es de 77 años, alrededor de 8 años más que los hombres, de 69 años. Se estima que estas cifras siguen aumentando, lo que requiere la atención de los servicios de salud para responder a las necesidades generadas por tal perfil demográfico, ya que la tercera incrementa la demanda de atención de salud ${ }^{(3)}$.

Las mujeres fueron responsables de grandes cambios porque viven más que los hombres, son la mayoría del electorado, tienen un mayor nivel de educación y son la mayoría en la Población Económicamente Activa con más de 11 años de estudios. Ellas están haciendo la transición de la exclusión a la participación ${ }^{(5)}$. 
No hay estudios epidemiológicos poblacionales realizados en mujeres brasileñas, y los estudios nacionales sobre la edad de la menopausia se estimaron en sólo las mujeres que asisten a los servicios médicos y por lo tanto no se consideran representativos de la población general. Existen pocos datos sobre estos temas en las poblaciones de América Latina, que difieren en el estilo de vida y los hábitos reproductivos de las poblaciones de los países desarrollados. Se cree que el conocer mejor la edad promedio de la menopausia y las características sociodemográficas y reproductivas de las mujeres menopáusicas, los servicios de salud serán capaces y estarán preparados para satisfacer las necesidades de estas mujeres, ya sea a través de una orientación adecuada, apoyo psicológico o alternativas diagnósticas y terapéuticas indicadas en diferentes situaciones ${ }^{(6)}$.

El término envejecimiento se utiliza a menudo para describir los cambios morfológicos y funcionales que se producen a lo largo de la vida, después de la maduración sexual y que socavan progresivamente la capacidad de respuesta de los individuos al estrés ambiental y mantener la homeostasis. Aunque no todas las funciones corporales sufren cambios significativos con el envejecimiento, es necesario recordar que este proceso tiene como una característica universal la ocurrencia de cambio en el tiempo, independientemente de si tienen un efecto perjudicial sobre la vitalidad y la longevidad. El envejecimiento no es necesariamente una suma agregada de patologías ${ }^{(7)}$, pero la probabilidad de su ocurrencia en la vejez se hace más grande.

Contrariamente a lo que pueda parecer, el envejecimiento no es patológico y, por tanto, debe ser visto como una etapa natural del desarrollo humano, como cualquier otro. Se caracteriza principalmente por disminución de la reserva funcional de todo el organismo, debido a un proceso degenerativo, progresivo e irreversible que da como resultado la reducción de la capacidad de adaptación homeostática, cuando se enfrentan con sobrecarga funcional ${ }^{(8)}$.

Cabe señalar que el proceso de envejecimiento humano no es homogénea y está influido por una serie de aspectos, por ejemplo, el género, el origen étnico y las condiciones socioeconómicas de las poblaciones ${ }^{(9)}$.

El envejecimiento puede ser biológico o psicológico. El envejecimiento biológico es implacable, activo e irreversible, causando más vulnerabilidad del organismo a los ataques externos e internos. Durante el envejecimiento, no es común la aparición de cambios en la función mental en los ancianos, o sea, los adultos mayores sanos y sin limitaciones físicas pueden ser bastante productivos. La definición de cuáles y cómo las funciones psíquicas son modificadas en el transcurso de los últimos años permite la consideración de que las personas mayores no sean tratadas como un ser limitado cognitivamente, sino que requiere la adaptación de los estímulos ambientales para tener funcionalidad comparable a la de los adultos jóvenes ${ }^{(10)}$.

Con el envejecimiento puede ocurrir una lentitud motora y cognitiva, así como deficiencias en las funciones sensoriales. Para que las personas mayores pueden participar en las conversaciones y tener la oportunidad de expresarse, las personas deben interactuar hablando lentamente y con la cara vuelta hacia ella ${ }^{(11)}$.

Se sabe que el envejecimiento sumado a las limitaciones de la actividad tiende a ralentizar la ejecución de las actividades diarias y que supervisar a los ancianos durante estas actividades requiere tiempo y paciencia para aquellos que la ven. Sin embargo, alentar a las personas mayores a desarrollar su potencial de independencia 
es una intervención que puede extender los años de vida y prevenir hospitalizaciones de salud y lesiones causadas por la inmovilidad y el estilo de vida sedentario con seguridad $^{(12)}$.

Los principios de un envejecimiento con calidad de vida describen a anciano como proactivo definiendo sus metas y luchando por alcanzarlas, reuniendo los recursos que son útiles en la adaptación al cambio y participando activamente en el mantenimiento del bienestar. Este modelo comprende desde la satisfacción con la vida a los modelos basados en conceptos de independencia, control, habilidades sociales y cognitivas ${ }^{(13)}$.

El Ministerio de Salud de Brasil en relación con la Política Nacional de Salud para la Tercera Edad, explica el concepto de la capacidad funcional como la capacidad de mantener las habilidades físicas y mentales necesarias para la vida independiente y autónoma $^{(14)}$.

Según Thober, Creutzberg, Viegas ${ }^{(15)}$ el anciano se considera saludable cuando no requiere "ningún tipo de ayuda o supervisión para realizar sus actividades diarias, pero que tiene una o más enfermedades crónicas." En la gerontología, la funcionalidad es el indicador más adecuado para dirigir la atención a los ancianos.

El envejecimiento se describe a menudo como un tiempo caracterizado por un agotamiento de las reservas, debido a las múltiples pérdidas que ocurren simultáneamente o en sucesión en un corto período de tiempo. Eventos negativos no normativos y graduados por edad, como la muerte de un cónyuge y amigos, deterioro de la salud y el funcionamiento físico, pérdida de estatus social y prestigio y, a veces, la inseguridad económica, además de la proximidad creciente de la muerte, se vuelven predominantes en la vejez avanzada. De hecho, algunos eventos raros o no normativos en las etapas anteriores (por ejemplo, enfermedad) se vuelven relativamente normativos en la vejez ${ }^{(16)}$.

Actualmente, se reconoce que la menopausia no puede ser entendida como un rango fisiológico de único evento. El período de transición del climaterio culminando en la menopausia es muy variable entre las diferentes culturas o dentro de la misma cultura. En las sociedades occidentales es una época conocida como de vulnerabilidades. El complejo hormonal psico-socio-cultural y los factores que vienen con la edad, a largo plazo, producen síntomas y diversos efectos sobre la salud. Muchas veces ocurre la salida de los niños de casa (el síndrome del nido vacío), la prima de jubilación, disminución de la líbido, la sensación de envejecimiento y la infertilidad, la aparición de enfermedades y depresión ${ }^{(17)}$.

En las sociedades de Oriente, la menopausia es un evento de reconocimiento de sexo femenino, ya que asocian este incidente con el envejecimiento y la atribuyen a ganar experiencia y sabiduría ante la vida. El no valorar en exceso la juventud y la belleza, difieren fundamentalmente de las mujeres occidentales. Así, la menopausia es vivda con más ligereza por las orientales. ${ }^{(18)}$.

En conjunto, estas perspectivas de riesgo y los desafíos sugieren que se requiere un aumento de la capacidad disponible y la capacidad de recuperación en la vejez para que el funcionamiento adaptativo se pueda mantener ${ }^{(19)}$.

La resiliencia tiene varias definiciones, revelando que el concepto está en 
construcción y debate. Originalmente, el término tiene la capacidad de recuperación física y se refiere a "la propiedad por la cual la energía almacenada en un cuerpo deformado es devuelta cuando cesa la tensión causadora de la deformación elástica" (20). La metáfora más evocada es la de elástico que, después de una tensión inicial, vuelve al mismo estado. Sin embargo, en relación con el ser humano, la capacidad de recuperación no significa un retorno a un estado anterior, sino la superación (o adaptación, como quieren algunos autores) ante una dificultad considerada un riesgo y la posibilidad de construir nuevas formas de vida y de un proceso de subjetivación a partir del enfrentamiento de las situaciones de estrés y / o traumáticas. Por lo tanto, sería una capacidad (singular y también adquirida socialmente) para hacerlo bien frente a actores potencialmente estresantes ${ }^{(21)}$.

Los factores de riesgo son los acontecimientos vitales que aumentan la probabilidad de que el individuo tenga problemas en su desarrollo. Ejemplos de este tipo de eventos son la muerte de un ser querido, la violencia física y psicológica, la enfermedad, la desintegración familiar, etc. ${ }^{(22)}$. Los factores de protección presentan como característica la posibilidad de disminución o reversión de uno o más factores de riesgo.

Factores ${ }^{(23)}$ protección tiene cuatro funciones principales: reducir el impacto de los riesgos; cuando el individuo está expuesto a una situación adversa; reducir las reacciones negativas en cadena después de la exposición al riesgo; establecer y / o mantener la autoestima y la autoeficacia, establecer relaciones de apego seguro, y crear oportunidades para revertir los efectos del estrés.

Los seres humanos tienen un límite para hacer frente al estrés, se sugiere que más de cuatro eventos estresantes de la vida ya son potenciadores de una condición de riesgo para el ser humano. Sin embargo, cómo el individuo experimenta una determinada situación parece ser más importante que el número de eventos estresantes experimentados por él ${ }^{(23)}$.

La vulnerabilidad puede entenderse como una predisposición que una persona tiene para desarrollar diversas formas de comportamiento no asertivo, así como una susceptibilidad a un resultado negativo en el desarrollo. Enfrente está la resiliencia, como la habilidad individual para tolerar las consecuencias negativas del riesgo, desarrolladas de forma adecuada ${ }^{(23)}$.

Una persona resiliente tiene características específicas, tales como tener un sentido de confianza en sí mismo, la creencia en su capacidad personal y la capacidad para resolver problemas ${ }^{(24,25)}$.

Ante las pérdidas, el self muestra la capacidad de recuperación o reserva de capacidad, es decir, el potencial para el mantenimiento y restauración de los niveles normales de adaptación y la presencia de rasgos latentes se puede activar a los retos y necesidades ${ }^{(26)}$. Se propone que las personas mayores, incluso en condiciones de limitaciones y discapacidades funcionales, mantengan un sentido positivo de bienestar ${ }^{(27)}$. Pérdidas de funcionalidad no son necesariamente un impedimento para la continuidad del funcionamiento cognitivo y emocional; como cualquier ser humano, los ancianos pueden activar mecanismos de compensación para hacer frente a estas pérdidas ${ }^{(28)}$.

Las personas mayores con amplia experiencia de vida tratan de distanciarse y 
reevaluar la situación positivamente, y buscar apoyo social. La función de la capacidad de recuperación es ayudar al ser humano a reformar sus comportamientos, a renovar sus actitudes ante las adversidades, a comprender los recursos disponibles, para buscar apoyo, buscando ganar cada desafío y aprender de cada lección. Se refiere a las respuestas que damos a los conflictos de la vida, la adversidad, a algún trauma en presencia de amenazas o riesgos. Digamosque es lo que llamamos nuestra fuerza interior, nuestra capacidad de resistir a los obstáculos del curso de la vida. Es ella la que da fuerza al individuo a mantener y preservar la estructura básica de su vida y a continuar ${ }^{(29)}$.

Todo el mundo se enfrenta a situaciones de estrés durante toda la vida, desde el nacimiento hasta la muerte, que puede traer varias consecuencias para la salud física y mental. Los tipos de eventos experimentados a lo largo de la vida varían de acuerdo a la edad cronológica, al tiempo histórico, con aspectos educativos y debido a factores individuales ${ }^{(29)}$.

Cuando hablamos de comportamiento, la resiliencia significa la construcción de nuevas formas de vida para hacer frente a situaciones muy estresantes o traumáticas.

La resiliencia no es sólo un rasgo hereditario que se tiene o deja de tener, es un logro personal.

En cuanto a la exposición a factores de riesgo, la activación de estrategias destinadas a la optimización, prevención y reparación es a menudo posible para alcanzar el nivel de adaptación. La activación se produce a menudo en condiciones sociales y culturales específicas, con el apoyo de la familia, lo que permite a los ancianos mantener su nivel de bienestar.

Nuestra capacidad de resiliencia tiene la función de ayudarnos a reformar nuestro comportamiento, renovando nuestras actitudes ante la adversidad, para superar todos los retos y aprender de cada lección. La resiliencia permite enriquecer nuestro curso de la vida, así como nuestra comprensión y los recursos disponibles, haciendo uso de nuestro repertorio de estrategias para lograr afrontar un envejecimiento exitoso. Sabiduría, las creencias positivas, la motivación, la autoconfianza reflejan este efecto de aprendizaje durante toda la vida.

Los factores de resiliencia son:

- Mantener una dieta saludable.

- Mantener la producción de endorfinas para la calidad de vida, a través de la risa, la buena respiración profunda y completa, ejercicios físicos, la relajación y la buena calidad de sueño contribuye a la producción de endorfinas.

- Administración de las emociones es la capacidad de mantener la calma ante un problema. Y también la capacidad de utilizar las pistas y "leer" a otras personas, para saber qué hacer.

- Control de los impulsos, no se deje llevar por la emoción impulsivamente.

- Optimismo. Es la creencia de que las cosas pueden mejorar. Es la esperanza y la fe en la capacidad de controlar su destino. 
- Análisis Ambiental. Es la capacidad de identificar las causas de los problemas, esto permite que la persona se ponga en lugar seguro. Saber dentificar cuándo hablar y cuándo callar.

- Empatía. Significa la capacidad de entender los estados psicológicos de los demás (emociones y sentimientos) y saber cómo actuar con las personas.

- Auto eficacia. Es la creencia de que puede resolver sus problemas.

- Llegar a la gente. Es la posibilidad de enlazar con otras personas, sin temor y sin miedo. Es la capacidad de encajar con los demás, construir redes de apoyo.

- Disciplina. El problema no está en la realidad sino en la forma en que respondemos a esta realidad. El problema no son las cosas que te pasan, el problema es lo que haces con las cosas que te pasan.

- Auto-confianza. Esta es la mayor característica del comportamiento resiliente. La superación sólo ocurre cuando en primer lugar, usted acredita su potencial, su capacidad de actuar positivamente.

Teniendo en cuenta las diversas definiciones, podemos entender la resiliencia, o como "una capacidad global de la persona para mantener un frente de trabajo efectivo contra la adversidad del medio ambiente o para la recuperación de estas condiciones", o como "un buen ajuste en las tareas de desarrollo de una persona, como resultado de la interacción entre el sujeto y la adversidad del medio ambiente o la participación de riesgo constante" ${ }^{(30)}$.

En la enfermería, la resiliencia comenzó a abordarse desde la década de 1990 con un mayor enfoque en las revistas americanas y europeas en las que las publicaciones se relacionan con las áreas de salud mental, pediatría, enfermería clínica y administración de enfermería. Sin embargo, en Brasil hay escasez de producción científica y existe una brecha el uso de este concepto en la enfermería gerontológica ${ }^{(31)}$.

Así que en la atención de enfermería, el diagnóstico riesgo de resiliencia comprometida comprende riesgo de disminución de la capacidad para mantener un patrón de respuesta positiva a una situación o crisis adversa, los factores de riesgo son cronicidad de las crisis existentes, múltiples situaciones adversas que coexisten y la presencia de más de una crisis (el embarazo no planificado, la pérdida de empleo, enfermedad, muerte en la familia) ${ }^{(32)}$

Considerando que con los años las personas de edad se vuelven más susceptibles a la angustia, el sufrimiento y la desesperanza, marcadas por el declive funcional, aislamiento, depresión y pérdida de autonomía, existe la necesidad de investigar el comportamiento de las mujeres menopáusicas y la resiliencia, tratando de entender si es que estos aspectos tienen algo en común, además de otros aspectos relacionados..

Por lo tanto, la identificación de los factores asociados a la resiliencia, permite comprender cuáles de ellos pueden ayudar a minimizar los efectos de los eventos estresantes en las personas mayores y promover un envejecimiento exitoso. 


\section{OBJETIVOS}

Analizar la capacidad de resiliencia de las mujeres mayores, las actividades de los participantes en el Centro Social de las ciudades del Valle de Paraíba, São Paulo, Brasil, variables sociodemográficas, edad, educación (años de educación formal), ingreso familiar, composición familiar, estado civil (casados, viudos, separados o solteros), práctica religiosa, cuidados de la salud física y mental (consumo de medicamentos), comorbilidades relatadas y su relación con el conjunto de actividades rutinarias, actividades sexuales, de ocio, profesional (rendimiento trabajo remunerado o no) y la asociación entre los niveles de resiliencia y las variables sociodemográficas, de salud y actividad física realizada.

\section{MATERIAL Y MÉTODO}

La investigación fue exploratoria, descriptiva, transversal, con un enfoque cuantitativo.

El estudio se realizó en los Centros Sociales de Mayores Valle de Paraíba, en São Paulo, Brasil. Las cuatro ciudades se eligieron por conveniencia del investigador.

Brevidelli, Domenico, aclaran en el muestreo de conveniencia, que está formada por miembros de la población que tienen mayor disponibilidad para la investigación o a las que el investigador tiene fácil acceso ${ }^{(33)}$.

El Valle de Paraíba es una región socioeconómica que ocupa parte del estado de São Paulo y el estado de Río de Janeiro, y que se destaca por concentrar una parte considerable del PIB. El nombre se debe al hecho de que la región es la parte inicial de la cuenca del río Paraíba do Sul. Debe tenerse en cuenta que el nombre se refiere sólo a una región con ciertas características socioeconómicas, ya que el río Paraíba do Sul se extiende por casi toda la extensión del estado de Río de Janeiro y separa parte de este estado de Minas Gerais. Su ubicación es en las orillas de la autopista Presidente Dutra, exactamente entre las ciudades de Río de Janeiro y Sao Paulo. La población sumada de todas las ciudades de la región es de casi 3,3 millones de habitantes ${ }^{(3)}$.

La población anciana total de las cuatro ciudades es 28.712. Se establecieron criterios de inclusión y exclusión para la selección de las personas mayores que podrían participar en el estudio, que se tradujo en 2.014 individuos. Para la recolección de datos se llevó a cabo el muestreo y cálculo computacional con significación del 5\%, lo que resulta en el número de 117 personas de edad avanzada, que compuso la muestra de esta investigación.

Este proyecto fue aprobado por el Comité Ético de Investigación (Plataforma Brasil) bajo n.383.699 en 09/11/2013.

Entre los participantes fueron incluidos en el estudio aquellos que cumplen con los criterios de inclusión, de edad inferior a 60 años, posmenopáusica, haber participado en las actividades estéticas y de bienestar hace seis meses, participantes del $\mathrm{CCl}$ hace un año que que constituyeron el campo de búsqueda, capaces de responder a los instrumentos de recolección de datos y que aceptaron participar como voluntarios, firmando Término de Consentimiento libre y esclarecido. 
Los criterios de exclusión fueron las mujeres que no participaron en las actividades de estéticas y las actividades CCl durante al menos un año, con edad inferior a 60 años, en desacuerdo en participar del estudio.

La recolección de datos se realizó en enero de 2014, seis meses después se organizaron conferencias informativas de resiliencia, actividades estéticas (facial, maquillaje, manicura, pelo, masaje corporal) degustación de frutas y jugos, finalizó con un espectáculo de danza del vientre para ayudar a desarrollar su autoestima y confianza en sí mismas.

Se utilizó como instrumento de recolección de datos, Escala de Resiliencia para evaluar los niveles de adaptación psicosocial positiva, cara a acontecimientos vitales, importantes de las encuestadas, versión adaptada para el portugués de laEscala de Resiliencia ${ }^{(30)}$. Hay 25 items descritos positivamente con la respuesta Likert que va de 1 (muy en desacuerdo) a 7 (muy de acuerdo). Las puntuaciones de la escala oscilan desde 25 hasta 175 puntos, los valores más altos indican una alta capacidad de recuperación.

Los datos fueron analizados estadísticamente de forma descriptiva, con un enfoque en la capacidad de recuperación.

Para comparar las variables categóricas entre los grupos se utilizó el ensayo de Medios - ANOVA (análisis de varianza) para comprobar si hay asociación entre el elemento en cuestión y la variable explicativa (edad, estado civil, educación, ingreso familiar, composición de la familia, religión, El uso de medicamentos, enfermedades, actividades de ocio y sexual).

\section{RESULTADOS Y DISCUSIÓN}

El estudio se caracteriza por mujeres con una media de edad de 70 a 80 años, viudas, católicas, jubiladas, 1-5 años de educación escolar, ingreso familiar de 2-3 salarios mínimos, actividad física, composición familiar 1-2 miembros en la familia, con percepción positiva para el cuidado de la salud física y mental, consumo de 1-2 tipos de medicamentos al día, tener 1-2 comorbilidades, informó la actividad sexual de 1 a 4 veces al mes y nivel de alta resiliencia.

En un estudio ${ }^{(34)}$ sobre actividad física, el mantenimiento de la capacidad funcional de los ancianos mostró que los programas de actividad física son los principales aspectos para el mantenimiento de la capacidad funcional.

Sobre la práctica de la actividad física en el Valle de Paraíba encontraron un predominio de mujeres de edad, solteras, con una edad media de 72 años, que además de formar parte del grupo, realiza una o más actividades físicas buscando siempre la promoción de la salud, ya sea por indicación médica o por iniciativa propia (35).

Resiliencia investigada en 546 ancianos en cinco centros de salud de Florianópolis, con edad de 75 años, sexo masculino, con vida social, educación superior, con mayor poder adquisitivo, independencia en las actividades instrumentales de la vida diaria, niveles más altos de actividad física, tenían valores más altos de resiliencia ${ }^{(36)}$. 
En las sociedades pacíficas, la cantidad de hombres y mujeres es casi igual hasta los 45 años; a partir de ahí, los hombres mueren a un ritmo más rápido, de modo que a los 70 años, hay cerca de seis mujeres por cada cinco hombres y a los 80, esta proporción es de cuatro por un anciano ${ }^{(37)}$.

Tabla 1 - Distribución de las mujeres participantes $(N=117)$ de acuerdo con la edad, el estado civil, la educación y la profesión. Valle de Paraíba, São Paulo, Brasil. 2014.

\begin{tabular}{l|l|l}
\hline Variables & \multicolumn{2}{|c}{$\begin{array}{l}\text { Participantes de edad } \\
\text { avanzada }\end{array}$} \\
\hline Edad & N & $\%$ \\
\hline $60-70$ años & 33 & 28,2 \\
\hline $70-80$ años & 69 & 59 \\
\hline $80-90$ años & 15 & 12,8 \\
\hline Estado civil & & \\
\hline Viuda & 53 & 45,3 \\
\hline Casado & 44 & 37,6 \\
\hline Soltero/ Independiente & 20 & 17,1 \\
\hline Años de educación & & \\
\hline $1-5$ años & 91 & 77,8 \\
\hline 5-10 años & 20 & 17,1 \\
\hline $10-15$ años & 6 & 5,1 \\
\hline
\end{tabular}

En cuanto al ingreso familiar $53(45,3 \%)$ de los encuestados mayores recibió entre 2.3 salarios mínimos mensuales, $39(33,3 \%)$ entre $1-2$ salarios y $25(21,4 \%) 4.3$ salarios, todas las personasmayores recibieron el beneficio del INSS.

Carlos et al. explica que las pensiones tienen un valor bajo, pero también implica en otras cuestiones de orden subjetivo, son las principales razones para mantener un vínculo con el trabajo y las actividades permeada por el deseo de reconocimiento y de permanecen sintiéndose útil ante una sociedad basada en el capitalismo ${ }^{(38)}$.

La educación es considerada un factor protector asociada con el bienestar psicológico, ya que cuantos más años de estudio de la materia, mayores son las destrezas y habilidades de esa persona en la medida de satisfacción con la vida y el equilibrio de los afectos ${ }^{(39)}$.

La composición de la familia estaba en 74 (63,2\%) de 1-2 personas, 24 (20,5\%) de 34 personas y $19(16,2 \%)$ de 5-6 personas.

Y la religión $96(82,1 \%)$ eran católicos, 14 (12\%) evangélica, 7 (6\%) informaron de ninguna religión.

Para Viktor Frankl ${ }^{(40)}$, el hombre religioso es aquel que fue capaz de completar su dinámica ontológica. Es responsable y consciente, vive su vida como una misión que debe cumplir. Así que en la búsqueda de sentido, se coloca la experiencia religiosa ${ }^{41)}$. La persona que busca una religión, encuentra los valores que le dirigen a una relación con lo que él considera ser "el creador", y esto, por lo tanto, hace que esté abierta a los demás y a la trascendencia.

En tiempos de sufrimiento, lo que se requiere de la persona es que soporte la capacidad de comprender racionalmente que la vida tiene un sentido incondicional, 
independientemente de las circunstancias. Este sentido incondicional se llama suprasentido. Sólo es aprendido por la fe, la confianza, por el amor, no puede ser comprendido por el razonamiento que responde a la pregunta de "¿por qué?" (40).

Para el cuidado de la salud física y mental $94(80,3 \%)$ de las encuestadas mayores reportaron una percepción positiva de la salud y 23 (19,7\%), una percepción negativa de la salud.

Para autores es la percepción de apoyo social la que protege a las personas contra la desestabilización ${ }^{(42,43)}$. Por lo tanto, el apoyo social puede ser considerado de vital importancia para hacer frente a las adversidades y dificultades de la vida, el vínculo entre las personas y el sentimiento de pertenencia y de ser respetados en sus capacidades, limitaciones y necesidades llevan a las personas a redescubrir la esencia del sus condiciones de vida saludables ${ }^{(44)}$.

La relación entre el apoyo social y la resiliencia son referidas ${ }^{(45)}$ como que emanan de cada persona, de cada ambiente y dependerá, en la mayoría de los casos, de la acción humana y de que la cultura de resiliencia se tiene la posibilidad de la vivencia dentro de una "trasparencia psicosocial".

En cuanto al consumo de medicamentos $56(47,9 \%)$ de más edad utilizan 1-2 medicinas diarias, $39(33,3 \%)$ 3-4 medicamentos diarios, y 22 (18,8\%) 5-6 medicamentos diarios.

La media de fármacos utilizados por las personas de edad es de entre dos y cinco ${ }^{(46)}$ y parece aumentar a expensas de los medicamentos sin receta.

El envejecimiento es un factor de predicción del consumo de medicamentos y su efecto produce incluso antes de los 60 , debido a que la probabilidad de consumirlos aumenta a partir de la cuarta década de la vida ${ }^{(47)}$.

En Brasil, los estudios de población sobre el consumo de productos farmacéuticos muestran un aumento de su toma con la edad, tanto en el interior de los pequeños pueblos ${ }^{(48)}$, como en los grandes centros urbanos. El número medio de los productos consumidos oscila entre $2^{(49)}$ y $3,24{ }^{(50)}$. En Río de Janeiro, Brasil ${ }^{(51)}, 80,19 \%$ de las personas mayores utilizan regularmente los medicamentos con receta, este uso es más acusado entre las mujeres y después de los 70 años de edad. Casi el 30\% toma medicamentos sin receta, sin diferencias entre los sexos, siendo la mayoría de los consumidores más jóvenes de entre 60 y 69 años.

Además de la edad y el sexo, otros factores predisponentes para el consumo de drogas se han identificado en el país. En el grupo de mujeres de edad avanzada con un mayor ingreso familiar y con más síntomas utilizan los medicamentos más recetados. El tamaño de la familia y favorables actitudes hacia los servicios no médicos son variables disponibles para las ventas de productos de consumo sin receta ${ }^{(50)}$.

Las comorbilidades fueron la hipertensión y las enfermedades cardiovasculares, 57 $(48,7 \%)$ tenían enfermedad 1-2, $34(29,1 \%)$ 3-4 enfermedad, $19(16,2 \%)$ de 5-6 enfermedades y $7(6 \%)$ ninguna. 
Una enfermedad es causa principal de sufrimiento, a menudo destruyuendo las estructuras más profundas de la vida. Aliviar el sufrimiento de la persona enferma constituye el deber ético y práctico de las profesiones incluidas en la salud, guiadas por el concepto de humanización de la atención ${ }^{(52)}$.

Comprender y aliviar el sufrimiento humano debe ser entendido como un elemento fundamental de la atención. Los estudios han demostrado que la enfermedad física puede llevar a situaciones de desequilibrio psicológico, se considera que los aspectos psicológicos y sociales de la enfermedad y la discapacidad se extienden a las bases de la conducta humana, lo que lleva al hombre a los cambios en el proceso de adaptación ${ }^{(53)}$.

La actividad física para $106(90,6 \%)$ de las mayores entrevistadas fue considerada como ocio y para las demás $11(9,4 \%)$ los viajes.

Un estudio ${ }^{(54)}$ constató que la participación de las mujeres mayores en un programa de actividad física llevó a beneficios psicológicos, como sentirse feliz con una misma, y ampliar sus redes sociales. Encuesta realizada con holandesas, con edades comprendidas entre 18 y 65 años en la práctica de ejercicio físico, como actividad de ocio y bienestar (satisfacción con la vida y la felicidad), encontró que en todas las edades, realizar el ejercicio fue asociado con mayores niveles de satisfacción con la vida y la felicidad ${ }^{(55)}$.

Investigación realizada ${ }^{(56)}$ con japoneses de edad avanzada al asociar los niveles de actividad física diaria entre los eventos estresantes, experimentados en el período de un año, encontraron que los acontecimientos estresantes parecen estar asociados con un bajo nivel de actividad física. Del mismo modo ${ }^{(57)}$, al investigar a ancianas brasileñas practicantes de actividad física, se encontró que la aparición de problemas emocionales como la muerte de niños, la enfermedad grave y dificultades financieras estaban relacionados con el nivel de estrés.

Una búsqueda ${ }^{(58)}$ cohorte con 544 ancianos pertenecientes al Estudio de Envejecimiento Saludable, en una ciudad de Minas Gerais, Brasil, identificó entre los principales eventos estresantes experimentados por los hombres y mujeres mayores de 60 años, los últimos cinco años, la muerte / pérdida y problemas salud de los ancianos. Según los autores, la investigación con personas mayores en diferentes situaciones, ya sea en la atención clínica o grupos sociales, ha puesto de manifiesto la preocupación de las personas mayores con pérdida de autonomía e independencia para realizar actividades diarias como consecuencia de la vejez, lo que favorece la aparición de enfermedades discapacitantes.

En cuanto a a actividad sexual $57(48,7 \%)$ de las ancianas informó practicar 1-4 veces al mes, $33(28,2 \%) 5-8$ veces al mes y $27(23,1 \%)$ sin actividad.

En un estudio de 44 personas mayores del Centro de Vida en el Valle de Paraíba, São Paulo, Brasil, con predominio femenino, alfabetizados, con un ingreso mensual de hasta un salario mínimo, jubilados, activos físicamente, para la actividad sexual, 31 $(70,45 \%)$ informaron que no practican el sexo y $5(38,46 \%)$ tener sexo al menos una vez a la semana ${ }^{(59)}$.

Vale la pena señalar que las actitudes discriminatorias hacia el envejecimiento, a veces se expresan a través de las reacciones de abstinencia, asco y ridículo, por lo 
que, aún hoy en día, las personas mayores se ven como personas indefensas y sin valor ${ }^{(28)}$. Por no tener el perfil ideal establecido por la sociedad, las personas mayores a menudo se perciben como seres asexuados, y la expresión sexual resulta algo inapropiado o incluso prohibido para personas mayores.

Según Negreiros, el anciano con miedo de convertirse en ridículo y también para escapar del estigma de "viejo (a) sibilante" pasa a adoptar, en general, una postura discreta reprimiendo sus actitudes y deseos sexuales, lo que conduce a una pérdida en la calidad de vida de las personas de edad avanzada ${ }^{(60)}$.

La suspensión o involuntario abandono de la sexualidad puede acelerar el proceso de envejecimiento, el impacto adverso sobre la salud en general y dañar la calidad de vida de las personas mayores ${ }^{(61)}$.

Para Capodieci (62) Ias personas mayores tienen más tiempo para el amor, son capaces de concentrarse más en las relaciones sociales y emocionales, es posible que, en la vejez, las experiencias sexuales alcancen su nivel más maduro. Por lo tanto, la sexualidad en la vejez es fisiológicamente posible, emocional y afectivamente enriquecedora, fotaleciendo la importancia del afecto, el apego, la comunicación, el compañerismo y el cuidado mutuo ${ }^{(63)}$.

Escala de Resiliencia: los participantes de edad avanzada no tienen dificultades para responder a las preguntas del instrumento, siendo fácil y rápida para ser aplicado en el estudio de la resiliencia con personas mayores, dado que el tiempo promedio de la entrevista fue de 8 minutos. En cuanto a los resultados obtenidos de la escala, la puntuación media fue un indicador de 133,72, lo que indica una alta puntuación.

Estos resultados confirman la capacidad de resiliencia al revelar positivamente la forma en que los ancianos viven su vejez, al ser un facilitador de desarrollo de recursos y la lupa de las posibilidades de un buen envejecimiento, calidad de vida, incluso antes de los cambios significativos de la vida de las personas mayores ${ }^{(64)}$.

Luthar ${ }^{(16)}$ señala que el individuo es capaz de reverter situaciones adversas con actitudes positivas, volviéndose un sujeto más seguro y más estable ante la adversidad, inclusive aquellos inherentes a la experiencia de envejecimiento, pueden ser considerados resistentes. La resiliencia se entiende como un proceso dinámico de adaptación positiva en contextos de adversidad. Los pensamientos y comportamientos que ayudan al individuo a enfrentar, de una manera asertiva, situaciones difíciles de la vida, maximizan el proceso de resiliencia y se denominan factores de protección.

Estudio de 86 ancianos, 67 mujeres y 19 varones, en la región metropolitana de Porto Alegre, señaló que la media obtenida a través de la Escala de Resiliencia fue de 84,9, lo que demuestra que las personas mayores encuestadas fueron capaces de superar las adversidades que surgieron en su vida, sin embargo, debido a la resiliencia es una manera de sobrevivir relacionada con la integridad, la adaptabilidad y la tenacidad ${ }^{(65)}$.

Tienen Asociación Resiliencia por el valor p (ANOVA) las ancianas de 70-80 años, solteras / separadas, católicas, 5-10 años de educación escolar, ingreso familiar de 2.3 salarios mínimos, físicamente activas, toman 4-5 tipos de medicamentos diarios, 5-6 comorbilidades y actividad sexual 5-8 veces por semana. 
Un estudio ${ }^{(66)}$ con 1.719 participantes suecos de edades comprendidas entre 19 y 103 años investigó la resiliencia y su relación con la edad y el género, los resultados mostraron que la resiliencia se relaciona con la edad, presentando mayores índices los más viejos.

La resiliencia también se ha asociado ${ }^{(67)}$ con la intensidad percibida de los eventos estresantes y la autoestima, el uso de medicamentos, la religión y la percepción del estado de salud, personas mayores practicantes de ejercicio físico tienden a tener una mayor capacidad de recuperación, y esto está asociado con un mejor estado de ánimo y la percepción de la salud, una mayor autoestima, la felicidad y la educación, menor consumo de medicamentos y la intensidad percibida de los acontecimientos estresantes, estos son factores clave que pueden ayudar a las personas mayores para hacer frente de manera más positiva en situaciones de estrés.

\section{CONCLUSIÓN}

Ante los sujetos estudiados, las mujeres mayores en el Valle de Paraíba, podemos decir que la edad promedio fue de 78 años, viudas, católica, jubiladas, 1-5 años de educación escolar, ingreso familiar de 3.2 salarios mínimos, practicantes de actividad física, composición familiar de 1-2 miembros en la familia, con la percepción positiva para el cuidado de la salud física y mental, consumo de 2-3 tipos de medicamentos al día, tener 1-2 comorbilidades, reportó actividad sexual 1-4 veces al mes y con un alto nivel de resiliencia.

Las ancianas contestaron a las preguntas de la escala resiliencia de forma rápida y fácil de entender, la puntuación media fue de 133.72 que poseen Asociación Resiliencia por el valor $p$ (ANOVA) de mayor edad 70-80 años, solteras / separadas, católicas, 5-10 años de educación escolar, ingreso familiar de 2.3 salarios mínimos, actividad física, consumo de 4-5 tipos de medicamentos por día, 5-6 comorbilidades y actividad sexual de 5 a 8 veces por semana.

Es importante hacer hincapié en la necesidad de implementar programas destinados a restaurar y mantener la autoestima de las personas mayores, lo que proporciona una mejor calidad de vida. Estos programas deben proporcionar carácter multidisciplinar proporcionando a los ancianos la convivencia con las otras generaciones.

\section{REFERENCIAS}

1. Lloyd-Sherlock P. Population ageing in developed and developing regions: implications for health policy. Soc Sci Med; 2000, 51:887-95.

2. Gordilho A, et al. Desafios a serem enfrentados no terceiro milênio pelo setor saúde na atenção integral ao idoso. Rio de Janeiro: UnATI/UERJ; 2000.

3. IBGE - Instituto Brasileiro de Geografia e Estatística. Síntese de Indicadores Sociais Uma Análise das Condições de Vida da População Brasileira. Rio de Janeiro. 2010/2012.

4. Chaimowicz, F. A saúde dos idosos brasileiros às vesperas do século XXI: problemas, projeções e alternativas. Saúde Pública, 1997, 31(2): 184-200.

5. Alves, J.E.D., Cavenaghi, S., Barros, L.F.W. A família DINC no Brasil: algumas características sócio demográficas. Textos para discussão. Escola Nacional de Ciências Estatísticas. 2010. 30-34. 
6. Pedro, A. O. et al . Idade de ocorrência da menopausa natural em mulheres brasileiras: resultados de um inquérito populacional domiciliar. Cad. Saúde Pública, Rio de Janeiro. 2003, 19, n. 1 .

7. Jeckel-Neto, E.A.; Cunha, G.L. Teorias Biológicas do Envelhecimento. In: Freitas, Elizabete Viana de, PY, Ligia, Cançado, Flávio Aluizio Xavier, DOLL, Johannes, Gorzoni, Milton Luiz. Tratado de Geriatria e Gerontologia. Rio de Janeiro: Editora Guanabara Koogan, 2006. 13-22.

8. Carvalho Filho, E.T. Fisiologia do envelhecimento. In: Papaléo Netto, M. Gerontologia. São Paulo: Atheneu, 2006. 60-70.

9. Brasil, Ministério da Saúde. Envelhecimento e saúde da pessoa idosa. Cadernos de Atenção Básica, Brasília, DF, 2006.19.

10. Moraes, et al. Características biológicas e psicológicas do envelhecimento. Rev. Med. Minas Gerais. 2010. 20(1): 67-73.

11. Bertachini, L.; Gonçalves, M. de J. Comunicação na terceira idade. Mundo saúde. São Paulo, 2002. v. 26, n. 4, 483-489.

12. Machado, F.N. Capacidade e desempenho para a realização das atividades básicas de vida diária: um estudo com idosos dependentes. Dissertação (Mestrado em Enfermagem) Universidade Federal de Minas Gerais. 2010, 129.

13. Inouye, K.; Pedrazzani, E.S.; Pavarini, S.C.I. Implicações da doença de Alzheimer na qualidade de vida do cuidador: um estudo comparativo. Cad. Saúde Pública. Rio de Janeiro. 2010. 26, n.5.

14. Miranda, R.D. Atividade física e envelhecimento. São Paulo:UNIFESP, 2005.

15. Thober, E.; Creutzberg, M.; Viegas, K. Nível de dependência de idosos e cuidados no âmbito domiciliar. Revista Brasileira de Enfermagem. Brasília, 2005. v. 58, n. 4, p. 438-443.

16. Fortes, T.F. R.; Portuguez, M. W.; Argimon, I. I. L. A resiliência em idosos e sua relação com variáveis sociodemográficas e funções cognitivas. Estud. psicol. Campinas, 2009.v. 26, n. 4.

17. Amore M, Di Donato P, Berti A, Aldopaolo P, Chirico C, Papalini A et al. Sexual and psycological symptoms in the climateric years. Maturitas. 2007 mar; 56(2): 30111.

18. De Lorenzi DRS, Danelon C, Saciloto B, Padilha Júnior I. Fatores indicadores da sintomatologia climatérica. Rev. Bras. Ginecol. Obstet. 2005; 27(1).

19. Schaie, K. W., \& Hofer S. M. Longitudinal studies in research on aging. In J. E. Birren \& K. W. Schaie (Eds.), Handbook of the psychology of aging (pp.53-77). San Diego, CA: Academic Press. 2001.

20. Ferreira, A.B.H. Novo dicionário da língua portuguesa.Rio de Janeiro: Nova Fronteira. 1975.

21. Lindström, B. O significado de resiliência. Adolescência Latino-Americana, 2001. 133-137.

22. Bianchini, D. C. S.; Dell'Aglio, D. D. Processos de resiliência no contexto de hospitalização: um estudo de caso. Paidéia. 2006. v. 16, n. 35, 427-436.

23. Pesce, R. P.; Assis, S. G.; Avanci, J. Q.; Santos, N.C.; Malaquias, J. V.; Carvalhaes, R.. Adaptação transcultural,confiabilidade e validade da escala de resiliência. Cad. Saúde Pública. Rio de Janeiro, 2005. 430 - 448.

24. Moreno-Jiménez, Bernardo. Psicología de la personalidad: procesos. Madrid:

Thomson, 2007.

25. Pinheiro, Débora Patrícia Nemer. A resiliência em discussão. Psicologia em estudo, Maringá. 2004. v. 9, n. 1, 67-75.

26. Staudinger, U..M., Marsiske, M., \& Baltes, P..B. Resiliência e níveis de capacidade de reserva na velhice: perspectivas da teoria de curso de vida. In A..L. Neri (Org.), 
Psicologia do envelhecimento: temas relacionados na perspectiva de curso de vida. Campinas: Papirus. 1995. 195-228.

27. Smith, J., Borchelt, M., Maier, H., \& Jopp, D. Health and well-being in the young and oldest old. Journal of Social Issues, 2002. 58 (4), 715-732.

28. Neri, A. L. Velhice e qualidade de vida na mulher. In A..L. Neri (Org.), Desenvolvimento envelhecimento: perspectivas biológicas, psicológicas e sociológicas. Campinas: Papirus. 2001. 161-200.

29. Pesce, R., Assis, S. G., Avanci, J., Malaquias, J., Oliveira, R. V. C. Adaptação transcultural, confiabilidade e validade da escala de resiliência. Cadernos de Saúde Pública. 2005. 21 (2), 436-448.

30. Martins, M. Contribuições para a análise de crianças e jovens em situação de risco - resiliência e desenvolvimento. Tese de Doutoramento apresentada à Faculdade de Ciências Humanas e Sociais da Universidade do Algarve. 2005.

31. Soria, D.A.C., Santoro, D.C., Souza, I.E.O., Menezes, M.F.B. A resiliência como objeto de investigação na enfermagem. Esc. Anna Nery Rev. Enferm. 2006. 10(3):547-51.

32. North American Nursing Diagnosis Association-Internacional. Diagnósticos de enfermagem da NANDA: definições e classificação - 2009-2011. Porto Alegre: Artmed, 2010.

33. Brevidelli, M.M.; Domenico, E.B.L. Trabalho de Conclusão de Curso: Guia Prático para Docentes e Alunos da Área da Saúde. 1aㅡ Ed. São Paulo: látria, cap. 2, 2006.

34. Silveira, S.C.; Araújo, C.L.O; Mancussi e Faro, A.C,.Atividade Física, Manutenção da Capacidade Funcional e da Autonomia em Idosos: Revisão de Literatura e Interfaces do Cuidado. Estud. interdiscipl. envelhec., Porto Alegre, 2011.v. 16, 61-77.

35. Araújo, C.L., Manucussi e Faro A.C. Prática de atividade física entre idosos do Vale do Paraíba, São Paulo, Brasil. Enfermeria Global, 2012. v.28, 223-231.

36. Hardy, S.E.; Concato, J.; Gill, T.M. Resilience of community-dwelling older persons. Journal of the American Geriatrics Society. 2004.52 (2): 257-262.

37. Netto, M. P. O estudo da velhice no século $X X$ : histórico, definição do campo e termos básicos. In E. V. Freitas, P. .Y. Ligia, F..A..X. Cançado \& M..L. Gorzoni. Tratado de geriatria e gerontologia Rio de Janeiro: Guanabara Koogan. 2002. 2-12.

38. Carlos, S.A.; Jacques, M.G.C.; Larratéa, S.V.; Herédia, O. C. Identidade, Aposentadoria e Terceira Idade. Estud Interdiscip Envelhec. 1999.1(1), pp. 77-89.

39. Queroz, N.C.; \& Neri, A.L. Bem-estar psicológico e inteligência emocional entre homens e mulheres na meia-idade e na velhice. Psicologia: Reflexão e Crítica: 2005. 18(2), 292-299.

40. Frankl, V. E. Em busca de sentido: um psicólogo no campo de concentração. Petrópolis: Vozes. 2006.

41. Coelho Junior, A. G., \& Mahfoud, M. As dimensões espiritual e religiosa da experiência humana: distinções e inter-relações na obra de Viktor Frankl. Psicologia USP, 2001.12 (2), 95-103.

42. Brito, R. C. \& Koller, S. H. Desenvolvimento humano e redes de apoio social e afetivo. Em A. M. A. Carvalho (Org.), O mundo social da criança: natureza e cultura em ação. São Paulo: Casa do Psicólogo. 1999. 115-126

43. Tavares, J. (Org.) Resiliência e educação. São Paulo: Cortez.

44. Boog, G., \& Boog, M. Manual de gestão de pessoas e equipes: Estratégias e tendências. Vol. 1. São Paulo: Gente. 2002.

45. Célia, S. S. Risco e resiliência em adolescência; aspectos clínicos e psicossociais. Porto Alegre: Artes Médicas. 2001.

46. Stuck, A. E.; Beers, M. H.; Steiner, A.; Aronow, H.; Rubenstein, L. Z. \& BECK, J. C., Inappropriate medication use in community-residing older persons. Archives of Internal Medicine, 1994.154: 2195-2200. 
47. Bardel, A.; Wallander, M. \& Svärdsudd, K., Reported current use of prescription drugs and some of its determinants among 35 to 65 -year-old women in mid-Sweden: A population-based study. Journal of Clinical Epidemiology, 2000. 53:637-643.

48. Haak, H., Padrões de consumo de medicamentos em dois povoados da Bahia. Revista de Saúde Pública, 1989. 23:143-151.

49. Barros, M. B. A., Saúde e Classe Social: Um Estudo sobre Morbidade e Consumo de Medicamentos. Tese de Doutorado, Ribeirão Preto: Faculdade de Medicina de Ribeirão Preto, Universidade de São Paulo. 1983.

50. Miralles, M. A., Acess to Care and Medication Use Among the Ambulatory Elderly in Rio de Janeiro, Brazil. Ph.D. Thesis, Gainesville: University of Florida. 1992.

51. Veras, R. P., País Jovem de Cabelos Brancos. A Saúde do Idoso no Brasil. Rio de Janeiro: Editora Relume-Dumará. 1994.

52. Barros Rudnicki, T.M. Psicologia e Saúde: intervenção em hospital geral. Aletheia, 1999.10: 115-120.

53. Yunes, M.A.M., Garcia, N.M., \& Albuquerque, B.B. Monoparentalidade, pobreza e resiliencia: entre as crenças dos profissionais e as possibilidades de convivencia familiar. Psicol Reflex Crit, 2007.20(3): 444-453.

54. Bidonde, M.J.; Goodwin, D.L.; Drinkwater, D.T. Older women's experiences of a fitness program: the importance of social networks. Journal of applied sport psychology. 2009.21(Supl.1): 86-101.

55. Stubbe, J.H.; DE Moor, M.H.M.; Boomsma, D.I.; DE Geus, E.J.C. The association between exercise participation and well-being: a co-twin study. Preventive Medicine. 2007.44(2): 148-152;

56. Yoshiuchi, K; Inada, S.; Nakahara, R.; Akabayashi, A.; Park, H.; Park, S., et al. Stressful life events and habitual physical activity in older adults: 1-year accelerometer data from the Nakanojo Study. Mental Health and Physical Activity. 2010.3(1): 23-25.

57. Luft, C.B. Aspectos neuropsicológicos do envelhecimento e prática de atividade física: possíveis relações em mulheres idosas. [Dissertação]. Florianópolis, Universidade do Estado de Santa Catarina - Centro de Educação Física, Fisioterapia e Desportos, 2007.

58. Fortes-Burgos, A.C.G.; Neri, A.L.; Cupertino, A.P.F.B. Eventos de vida estressantes entre idosos brasileirosresidentes na comunidade. Estudos de Psicologia. 2009.14(1): 69-75.

59. Ferreira, K. S.; Silva, M. G.; Cherem, T. M. D. A.; Araújo, C.L. O. Percepção dos idosos perante o sexo na idade avançada. Geriatria \& Gerontologia.3(4):182188.2009.

60. Negreiros, T.C.G.M. Sexualidade e Gênero no envelhecimento. Alceu. 2004.5(.9), 77-86.

61. Pascual, C.P. A sexualidade do idoso vista com novo olhar. São Paulo: Loyola. 2002.

62. Capodieci , S. A idade dos sentimentos: Amor e sexualidade após os sessenta anos. Bauru: EDUSC. 2000.

63. Urquiza, A., Thumala, D., Cathalifaud, M.A., Ojeda, A., \& Vogel, N. Sexualidad em la tercera edad. La imagen de los jóvenes universitários. 2008.4, 358-374.

64. Koller, S. H. et al. Adolescentes e jovens em situação de risco psicossocial: redes de apoio social e factores pessoais de protecção. Estudos de Psicologia. 2008.13 (2), 165-174.

65. Luthar, S., Cicchetti, D., Becker, B. The Construct of Resilience: A critical evaluation and guidelines for future work. Child De velopment. 2000. 71 (3), 543-562. 66 . Lundman, B. Psychometric properties of the swedish version of the resilience scale. Scandinavian Journal of Caring Science. 2007. 21 (2), 229-237. 
67. Hardy, S.E.; Concato, J.; Gill, T.M.. Resilience of community -dwelling older persons. Journal of the American Geriatrics Society. 2004.53 (2): 257-262.

Recibido: 21 de diciembre 2014; Aceptado: 24 de enero 2015 